\title{
GW23-e0447 VASCULAR ENDOTHELIAL FUNCTION MEASUREMENT AND INSULIN RESISTANCE INDEX CONTRIBUTE TO THE PREDICTION OF ERECTILE DYSFUNCTION IN YOUNG MAN
}

doi:10.1136/heartjnl-2012-302920ad.9

Yao Fengjuan, Zhang Yan, Huang Yanping, Liu Donghong, Chen Yili, Lin Hong, Lu Kun, Fan Rui, Liu Yanqiu, Deng Chunhua, Zhang Yan. The First Affiliated Hospital of SUN YAT-SEN University

Objectives Investigate the relationship between glycometabolic disorders and erectile dysfunction (ED) without organic aetiology in young man under the age of 40 years.

Methods 192 patients and 33 normal controls were enrolled. ED was evaluated by using the International Index of Erectile Function- 5 (IIEF-5) questionnaire. We measured traditional cardiovascular risk factors, hormone levels and vascular parameters. The HOMA index was calculated as the product of the fasting plasma insulin level $(\mu \mathrm{U} /$ $\mathrm{ml}$ ) and the fasting plasma glucose level (mmol/l), divided by 22.5 . Insulin Resistance (IR) was measured by homeostasis model assessment (HOMA).

Results Patients with ED had significantly higher systolic blood pressure (SBP), High-sensitivity C-reactive protein (hsCRP), high Insulin resistance index (HOMA- IR) and carotid intima- media thickness (IMT), compared with controls. Brachial artery endothelium- dependent flow- mediated vasodilation (FMD) was significantly reduced in ED patients. By multivariate logistic regression analysis, FMD, SBP, hsCRP and HOMA- IR were significantly associated with ED. In receiver- operating characteristic (ROC) analysis, FMD was a significant predictor of ED (area under the curve (AUC) 0.928, p <0.001). The cut-off value of FMD $<9.6 \%$ had sensitivity of $80.9 \%$ and specificity of $100 \%$. HOMA- IR were also predictor of ED (AUC of HOMAIR $0.762, p<0.001$ ).

Conclusions ED may be the first clinical sign of endothelial dysfunction and a clinical marker of cardiovascular and metabolic diseases. Endothelial dysfunction, underlying insulin resistance in young ED patients without well-known related risk factors may be the underlying pathogenesis of ED in young patients as in elderly one. Measurement of FMD, HOMA- IR can improve our ability to predict $\mathrm{ED}$ in young man. 